LETTER TO THE EDITOR

doi:10.1017/\$1041610221002763

\section{Public preferences about who should be prioritized in receiving the COVID-19 vaccine: people with or without Alzheimer's disease?}

Ever since the global outbreak of SARS-CoV-2, the virus that causes COVID-19, there has been an unparalleled race toward the development of a safe COVID-19 vaccine. In early December 2020, the first COVID-19 vaccines were approved for use in humans (CDC, 2021). However, in many countries, including Israel, when the vaccine rollout began, there were not enough vaccines for everyone, making it necessary to prioritize their receipt (Cylus et al., 2021). COVID-19 vaccines were considered a scarce resource at the time. Indeed, one of the principles for prioritizing resources under conditions of scarcity is the utility criterion, according to which priority should be given to those people who can benefit to a greater degree from such scarce resources, including having a greater chance for recovery and having more years of life ahead of them (Emanuel et al., 2020). Hence, the question arises as to whether people with dementia, who are usually old and have multiple comorbidities and a high risk of mortality (Taudorf et al., 2020), should receive COVID-19 vaccines. And what about young people with dementia? I examined these questions among the Israeli public prior to the availability of vaccines.

I believe it is important to examine public attitudes even though decisions regarding COVID-19 vaccine allocation are not made by the public, as previous studies have shown that public attitudes play an important role in the prioritization process. They allow the development of a transparent and fair policy based on the principles of democracy, which can increase the public's confidence in the health system and government (Bruni et al., 2008; Han et al., 2021). Also, public involvement in this process is a good way to obtain public support and is described as one of the main factors in controlling the COVID-19 outbreak, including public adherence to restrictions and uptake of vaccines (Gollust et al., 2020).

I therefore conducted an online cross-sectional survey among 309 Israeli Jews aged 40 years and over. The sample was equally divided between men $(47.9 \%)$ and women $(52.1 \%)$, and between secular
$(51.1 \%)$ and religious (48.9\%) individuals. The mean age was 56 years $(\mathrm{SD}=10.23)$, and participants reported an average of 15 years of education $(\mathrm{SD}=15.13)$.

I presented a description to the participants of three hypothetical individuals: Moses, a 55-year-old man with Alzheimer's disease (AD); Jacob, an 80-year-old man with $\mathrm{AD}$; and Samuel, an 80 -year-old man with no cognitive decline. The three were described as married, having three children, living at home with their spouses, and diagnosed with diabetes and high blood pressure. I asked participants to choose who should be first/last to receive the COVID-19 vaccine.

Participants' preferences regarding who should receive the vaccine last were clear (see Figure 1). Of the three choices, most participants $(67.6 \%)$ chose the 80 -year-old with $\mathrm{AD}$ to be last. The total difference under the null hypothesis of equal distribution was significant, $\chi_{(2)}^{2}=164.87, \mathrm{p}<0.001$, whereas the difference between Moses and Samuel was not significant, $\chi_{(1)}^{2}=2.56, p=0.110$. Regarding preferences about who should be first, the results were less clear but still revealed a trend. About half the sample $(50 \%)$ selected the 80 -year-old with no cognitive decline, while $39 \%$ selected the 55 -year-old with $\mathrm{AD}$, to be first. Indeed, under the null hypothesis of equal distribution the total difference was significant, $\chi_{(2)}^{2}=76.97, \mathrm{p}<0.001$, and the difference between Samuel and Moses was also significant, $\chi_{(1)}^{2}=4.19, \mathrm{p}=0.041$.

These important findings raise concerns about discrimination against people with dementia, and especially older people with dementia specifically at a time when it has been necessary to decide which groups should receive life-saving resources first (such as vaccines or ventilators). Indeed, nonprofit organizations (such as Alzheimer's Europe) have warned against discriminating against people with dementia in receiving health services during this crisis, emphasizing that background characteristics including age and cognitive status should not be criteria for receiving these services (Alzheimer's Europe, 2020). Such discrimination runs contrary to basic human rights as well as society's moral duty to provide health services to all (Carrieri et al., 2020).

Based on previous studies on a related subject (Werner and Landau, 2020), I also examined the relation between participants' preferences and several factors associated with their preferences: sociodemographic characteristics, moral reasoning, $\mathrm{AD}$ variables (i.e. stigmatizing attitudes toward a person 


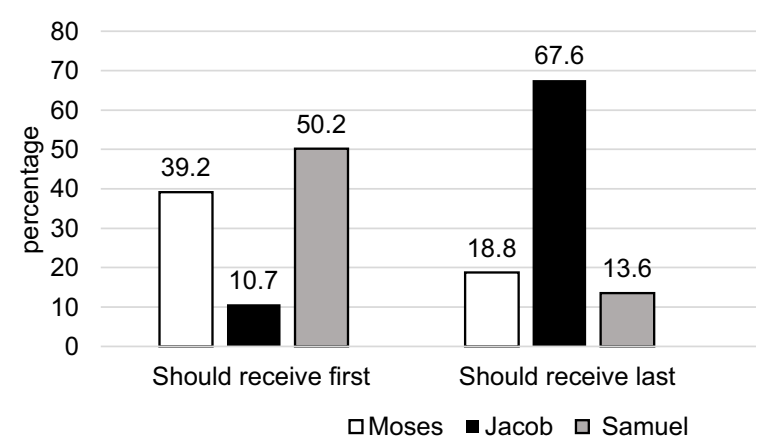

Figure 1. Participants' preferences for who should receive the COVID-19 vaccination first/last $(n=309)$. Moses: 55 -year-old with a diagnosis of AD. Jacob: 80-year-old with a diagnosis of AD. Samuel: 80-year-old, cognitively intact.

with $\mathrm{AD}$, beliefs about susceptibility to/fear of becoming ill with $\mathrm{AD}$, as well as subjective knowledge and familiarity with AD), and COVID-19 variables (i.e. beliefs about susceptibility to/fear of contracting COVID-19). Of these variables, only beliefs about susceptibility to contracting COVID19 , level of education, and familiarity with a person with $\mathrm{AD}$ were found to be significantly related to participants' preferences. Given the low number of statistically significant correlates found in this study, we would suggest future studies investigate other factors such as knowledge, perceptions, and attitudes concerning the COVID-19 vaccine.

Finally, information about public preferences in this context is important and should be available to policymakers so that they can make better decisions. Even if such decisions run contrary to public attitudes, understanding these attitudes will allow policymakers to develop interventions accordingly. Indeed, they may consider developing an intervention to reduce negative attitudes toward people with dementia. At the same time, they could prepare a protocol with instructions about how to allocate scarce resources specifically to people with dementia, to avoid exposing them to discriminatory responses. Such a protocol would also be helpful in guiding medical staff in making such decisions.

\section{References}

Alzheimer's Europe. (2020). Alzheimer Europe position regarding the allocation of scarce medical resources for intensive care services during the COVID-19 pandemic. Available at https://www.alzheimer-europe.org/Policy/
Our-opinion-on/Triage-decisions-during-COVID-19pandemic/(language)/eng-GB

Bruni, R. A., Laupacis, A. and Martin, A. K. (2008). Public engagement in setting priorities in health care. Canadian Medical Association fournal, 179, 15-18. DOI 10.1503/cmaj.071656.

Carrieri, D., Peccatori, F. A. and Boniolo, G. (2020). COVID-19: a plea to protect the older population. International fournal for Equity in Health, 72, 2663. DOI 10.1186/s12939-020-01193-5.

Center for Disease Control and Prevention (CDC). (2021). What older adults need to know about COVID19 vaccines. Available at https:/www.cdc.gov/ coronavirus/2019-ncov/vaccines/recommendations/ older-adults.html

Cylus, J., Panteli, D. and van Ginneken, E. (2021). Who should be vaccinated first? Comparing vaccine prioritization strategies in Israel and European countries using the Covid-19 Health System Response Monitor. Israel fournal of Health Policy Research, 10, 16. DOI 10.1186/s13584-02100453-1.

Emanuel, E. J., Persad, G., Upshur, R. et al. (2020). Fair allocation of scarce medical resources in the time of Covid-19. The New England fournal of Medicine, 382, 2049-2055. DOI 10.1056/NEJMsb2005114.

Gollust, S. E., Saloner, B., Hest, R. et al. (2020). US adults' preferences for public allocation of a vaccine for coronavirus disease 2019. fAMA Network Open, 3, e2023020. DOI 10.1001/jamanetworkopen.2020.23020.

Han, J. J., Shin, M., Patrick, W. L. et al. (2021). How should ECMO be used under conditions of severe scarcity? A population study of public perception. Fournal of Cardiothoracic and Vascular Anesthesia, 396, 1-8. DOI 10.1053/j.jvca.2021.05.058.

Taudorf, L., Nørgaard, A., Brodaty, H. et al. (2020). Dementia increases mortality beyond effects of comorbid conditions: a national registry-based cohort study. European fournal of Neurology, 28, 2174-2184. DOI 10.1111/ ene. 14875 .

Werner, P. and Landau, R. (2020). Laypersons' prioritysetting preferences for allocating a COVID-19 patient to a ventilator: Does a diagnosis of Alzheimer's disease matter? Clinical Interventions in Aging, 15, 2407-2414. DOI 10.2147/ CIA.S283015.

\section{Hanan AboJabel(1)}

Department of Community Mental Health, University of Haifa, Haifa, Israel

Correspondence should be addressed to: Hanan AboJabel, Ph.D., Department of Community Mental Health, University of Haifa, Mt. Carmel, Haifa, Israel. Phone: 972-55-6810001; Fax: 972-04-828-8723.

Email: hanan.abojabel.1987@live.com 